\title{
Correction to: Improvement of Renal Function After Bariatric Surgery: a Systematic Review and Meta-analysis
}

\author{
Hongyan Huang ${ }^{1,2,3} \cdot$ Jun $\mathrm{Lu}^{4} \cdot$ Xiaojiang $\mathrm{Dai}^{1,2} \cdot{\text { Zhixin } \mathrm{Li}^{5} \cdot \text { Liyong Zhu }^{6} \cdot \text { Shaihong Zhu }}^{6} \cdot$ Liangping Wu $^{1,2}$ \\ Published online: 7 September 2021 \\ ๑) Springer Science+Business Media, LLC, part of Springer Nature 2021
}

\section{Correction to: Obesity Surgery \\ https://doi.org/10.1007/s11695-021-05630-4}

Author Hongyan Huang and author Jun Lu are co-first authors. These authors (Hongyan Huang and Jun Lu) contributed equally.

Publisher's note Springer Nature remains neutral with regard to jurisdictional claims in published maps and institutional affiliations.

The original article can be found online at https://doi.org/10.1007/ s11695-021-05630-4.

Liangping $\mathrm{Wu}$

drwulp@163.com

Liyong Zhu

zly8128@126.com

Shaihong Zhu

shaihongzhu@126.com

1 Surgical Center for Obesity and Diabetes, Jinshazhou Hospital, Guangzhou University of Chinese Medicine, Guangzhou 510515, China

2 UDM Medical Group, Guangzhou 510515, China

3 Clinical Research Center, The Third Xiangya Hospital, Central South University, Changsha 410013, China

4 School of Medicine, Hunan Normal University, Changsha 410013, China

5 Clinical Medicine Eight-Year Program, Xiangya School of Medicine, Central South University, 18 Grade, Changsha 410013, China

6 Department of General Surgery, Third Xiangya Hospital, Central South University, Changsha 410013, China 